\title{
NEW EXAMPLES OF SASAKI-EINSTEIN MANIFOLDS
}

\author{
TOSHIKI MABUCHI* AND YASUHIRO NAKAGAWA**
}

\begin{abstract}
In this note, stimulated by the existence results [10] for toric Sasaki-Einstein metrics, we obtain new examples of SasakiEinstein metrics on $S^{1}$-bundles associated to canonical line bundles of $\mathbb{P}^{1}(\mathbb{C})$-bundles over Kähler-Einstein Fano manifolds, even though the Futaki's obstruction does not vanish. Here the method as in [23, [16], 18] is used, and our examples include non-toric Sasaki-Einstein manifolds.
\end{abstract}

\section{INTRODUCTION}

Sasaki-Einstein manifolds were studied not only by mathematicians but also by physicists, as Sasaki-Einstein manifolds have various interesting phenomena such as "AdS/CFT correspondence" in theoretical physics (cf. [2], [3], [4], [5], 6], [12], [19], [20], [21], [22]). Recently in [1] and [10], classification of toric Sasaki-Einstein manifolds was given.

A Sasaki manifold is a $(2 m+1)$-dimensional Riemannian manifold $(S, g)$ whose cone manifold $(C(S), \bar{g})$ is a Kähler manifold with

$$
C(S):=S \times \mathbb{R}_{>0} \text { and } \bar{g}:=(d r)^{2}+r^{2} g,
$$

where $r$ is the standard coordinate on the set $\mathbb{R}_{>0}=\{r>0\}$ of positive real numbers. Then $S$ is a contact manifold with the contact form

$$
\eta:=\left.(\sqrt{-1}(\bar{\partial}-\partial) \log r)\right|_{r=1} .
$$

Here $S$ is viewed as the submanifold of $C(S)$ defined by the equation $r=1$. We further consider the the Reeb field $\xi$ characterized by

$$
i(\xi) \eta=1 \text { and } i(\xi) d \eta=0,
$$

where $i(\xi)$ is the interior product by $\xi$. The Reeb field $\xi$ is a Killing vector field on $(S, g)$ with a lift to a holomorphic Killing vector field on $(C(S), \bar{g})$. This generates a 1-dimensional foliation on $S$, called the Reeb foliation. The Sasaki metric $g$ naturally induces a transverse Kähler metric $g^{T}$ for the Reeb foliation on $S$. A Sasaki manifold $(S, g)$ is toric, if $C(S)$ is a toric manifold.

The following well-known fact allows us to reduce the existence of Sasaki-Einstein metrics to that of transverse Kähler-Einstein metrics:

2010 Mathematics Subject Classification. Primary 53C25; Secondary 32Q20, $53 \mathrm{C} 55$.

*Supported by JSPS Grant-in-Aid for Scientific Research (A) No. 20244005.

**Supported by JSPS Grant-in-Aid for Scientific Research (C) No. 20540069. 
Fact 1.1 (cf. [4, Chapter 11]). A Sasaki manifold $(S, g)$ is Einstein with positive scalar curvature $2 m$ if and only if the transverse Kähler metric $g^{\mathrm{T}}$ is Einstein with positive scalar curvature $2(m+1)$.

We now pose the following conjecture:

Conjecture 1.2. Let $M$ be a Fano manifold. If there exists a KählerRicci soliton (see for instance [28] for Kähler-Ricci solitons) on $M$, then the $S^{1}$-bundle associated to the canonical line bundle $K_{M}$ of $M$ admits a Sasaki-Einstein metric with a suitable choice of the Reeb field.

By the results of Wang and Zhu [28], the existence of Kähler-Ricci solitons is known for toric Fano manifolds. Hence, the results in [10] shows that Conjecture 1.2 is affirmative for toric Fano manifolds.

We now consider Koiso-Sakane's examples [23], [16], [17] of $\mathbb{P}^{1}(\mathbb{C})$ bundles over Kähler-Einstein Fano manifolds. To fix our notation, recall the paper [18]. Under the assumption below, we fix once for all a compact connected $n$-dimensional complex manifold $W$ with $c_{1}(W)>0$ and an Hermitian holomorphic line bundle $(L, h)$ over $W$.

Assumption 1.3. (1) There exists a Kähler-Einstein form $\omega_{0}$ on $W$, i.e., $\operatorname{Ric}\left(\omega_{0}\right)=\omega_{0}$, where $\operatorname{Ric}\left(\omega_{0}\right)$ is the Ricci form for $\omega_{0}$.

(2) $2 \pi c_{1}(L ; h):=\sqrt{-1} \bar{\partial} \partial \log h$ has constant eigenvalues

$$
\mu_{1} \leqq \mu_{2} \leqq \cdots \leqq \mu_{n}
$$

with respect to $\omega_{0}$ satisfying $-1<\mu_{k}<1$ for $k=1,2, \ldots, n$.

By this assumption, the compactification $M_{W}^{L}:=\mathbb{P}\left(L \oplus \mathcal{O}_{W}\right)$ of $L$ is a $\mathbb{P}^{1}(\mathbb{C})$-bundle over $W$ with $c_{1}\left(M_{W}^{L}\right)>0$. Then $M_{W}^{L}$ admits a KählerEinstein metric if and only if its Futaki's obstruction (cf. [8]) vanishes:

$$
\int_{-1}^{1} x \prod_{k=1}^{n}\left(1+\mu_{k} x\right) d x=0 .
$$

Let $S_{W}^{L}$ be the $S^{1}$-bundle over $M_{W}^{L}$ associated to the canonical line bundle $K_{M_{W}^{L}}$ of $M_{W}^{L}$. In [15], Koiso showed that a Kähler-Ricci soliton exists on $M_{W}^{L}$, whether or not equality (1.4) holds. Hence by Conjecture 1.2, a Sasaki-Einstein metric is expected to exist on $S_{W}^{L}$. The purpose of this note is to give the following affirmative result:

Theorem 1.5. Under the Assumption 1.3, whether or not equality (1.4) holds, $S_{W}^{L}$ always admits a Sasaki-Einstein metric for a suitable choice of the Reeb field. Furthermore, $K_{M_{W}^{L}}$ admits a complete Ricci-flat Kähler metric in every Kähler class.

Remark 1.6. Kobayashi [14] (see also Jensen [13], Wang and Ziller [27]) constructed Einstein metrics on $S^{1}$-bundles over Einstein manifolds. Our theorem above shows that $S_{W}^{L}$ always admits an Einstein metric, even though $M_{W}^{L}$ admits no Kähler-Einstein metrics. 


\section{Transverse holomorphic structures on $S_{W}^{L}$}

For an open cover $\left\{U_{\alpha} ; \alpha \in A\right\}$ of $W$, we choose a system of holomorphic local coordinates $\left(w_{\alpha}^{1}, w_{\alpha}^{2}, \ldots, w_{\alpha}^{n}\right)$ on each $U_{\alpha}$, and by taking a holomorphic local frame $e_{\alpha}$ for $L$, we have the fiber coordinate $\zeta_{\alpha}^{+}$ for $L$ over $U_{\alpha}$ with respect to $e_{\alpha}$. Then $\left(w_{\alpha}^{1}, w_{\alpha}^{2}, \ldots, w_{\alpha}^{n} ; \zeta_{\alpha}^{+}\right)$forms a system of holomorphic local coordinates for $U_{\alpha}^{+}:=\left.L\right|_{U_{\alpha}}$. Let $f_{\alpha}$ be the frame for $L^{-1}$ dual to $e_{\alpha}$, and let $\zeta_{\alpha}^{-}$be the fiber coordinate for $L^{-1}$ over $U_{\alpha}$ with respect to $f_{\alpha}$. Then $\left(w_{\alpha}^{1}, w_{\alpha}^{2}, \ldots, w_{\alpha}^{n} ; \zeta_{\alpha}^{-}\right)$form a system of holomorphic local coordinates on $U_{\alpha}^{-}:=\left.L^{-1}\right|_{U_{\alpha}}$. Then $U_{\alpha}^{+}$and $U_{\alpha}^{-}$ are glued together by the relation

$$
\zeta_{\alpha}^{+}=\left(\zeta_{\alpha}^{-}\right)^{-1}
$$

to form $M_{W}^{L}=\mathbb{P}\left(L \oplus \mathcal{O}_{W}\right)=\bigcup_{\alpha \in A}\left(U_{\alpha}^{+} \cup U_{\alpha}^{-}\right)$. Here,

$$
\pm d w_{\alpha}^{1} \wedge d w_{\alpha}^{2} \wedge \cdots \wedge d w_{\alpha}^{n} \wedge d \zeta_{\alpha}^{ \pm}
$$

is a holomorphic local frame for $K_{M_{W}^{L}}$ over $U_{\alpha}^{ \pm}$, and with respect to this local frame, we have the fiber coordinate $\tau_{\alpha}^{ \pm}$for $K_{M_{W}^{L}}$, respectively, i.e., all (+)-signs and all ( - -)-signs should be chosen respectively. Note that

$$
\begin{aligned}
& \tau_{\alpha}^{+} d w_{\alpha}^{1} \wedge d w_{\alpha}^{2} \wedge \cdots \wedge d w_{\alpha}^{n} \wedge d \zeta_{\alpha}^{+} \\
& =\tau_{\beta}^{+} d w_{\beta}^{1} \wedge d w_{\beta}^{2} \wedge \cdots \wedge d w_{\beta}^{n} \wedge d \zeta_{\beta}^{+} \\
& =\tau_{\beta}^{+} \phi_{\beta \alpha}(w) \psi_{\beta \alpha}(w)^{-1} d w_{\alpha}^{1} \wedge d w_{\alpha}^{2} \wedge \cdots \wedge d w_{\alpha}^{n} \wedge d \zeta_{\alpha}^{+},
\end{aligned}
$$

for $w \in U_{\alpha} \cap U_{\beta}$. Here $\left\{\psi_{\beta \alpha} ; \alpha, \beta \in A\right\}$ are the transition functions for $L$ with respect to the local frames $\left\{e_{\alpha} ; \alpha \in A\right\}$ for $L$, while $\left\{\phi_{\beta \alpha} ; \alpha, \beta \in\right.$ $A\}$ are the transition functions for $K_{W}$ with respect to the local frames $\left\{d w_{\alpha}^{1} \wedge \cdots \wedge d w_{\alpha}^{n} ; \alpha \in A\right\}$ for $K_{W}$, i.e.,

$$
\begin{aligned}
& e_{\beta}=\psi_{\beta \alpha}(w) e_{\alpha}, \quad f_{\beta}=\psi_{\beta \alpha}(w)^{-1} f_{\alpha}, \\
& d w_{\beta}^{1} \wedge d w_{\beta}^{2} \wedge \cdots \wedge d w_{\beta}^{n}=\phi_{\beta \alpha}(w) d w_{\alpha}^{1} \wedge d w_{\alpha}^{2} \wedge \cdots \wedge d w_{\alpha}^{n},
\end{aligned}
$$

for $w \in U_{\alpha} \cap U_{\beta}$. Hence $\tau_{\alpha}^{+}$can be viewed as the fiber coordinate for $K_{W} \otimes L^{-1}$ over $U_{\alpha}$ with respect to the local frame $\left(d w_{\alpha}^{1} \wedge \cdots \wedge d w_{\alpha}^{n}\right) \otimes$ $f_{\alpha}$. Similarly, $\tau_{\alpha}^{-}$is also viewed as the fiber coordinate for $K_{W} \otimes L$ over $U_{\alpha}$ with respect to the local frame $\left(d w_{\alpha}^{1} \wedge \cdots \wedge d w_{\alpha}^{n}\right) \otimes e_{\alpha}$. Moreover, since $\tau_{\alpha}^{+} \zeta_{\alpha}^{+}=\tau_{\alpha}^{-} \zeta_{\alpha}^{-}$on $U_{\alpha}^{+} \cap U_{\alpha}^{-}$, it follows that

$$
\tau_{\alpha}^{+}\left(\zeta_{\alpha}^{+}\right)^{2}=\tau_{\alpha}^{-}
$$

Now, for $-\frac{1}{2}<a \in \mathbb{R}$, we consider holomorphic vector fields

$$
\begin{aligned}
& a \sqrt{-1} \zeta_{\alpha}^{+} \frac{\partial}{\partial \zeta_{\alpha}^{+}}+\sqrt{-1} \tau_{\alpha}^{+} \frac{\partial}{\partial \tau_{\alpha}^{+}} \text {on } \tilde{p}^{-1}\left(U_{\alpha}^{+}\right), \\
& -a \sqrt{-1} \zeta_{\alpha}^{-} \frac{\partial}{\partial \zeta_{\alpha}^{-}}+(1+2 a) \sqrt{-1} \tau_{\alpha}^{-} \frac{\partial}{\partial \tau_{\alpha}^{-}} \text {on } \tilde{p}^{-1}\left(U_{\alpha}^{-}\right),
\end{aligned}
$$

where $\tilde{p}: K_{M_{W}^{L}} \longrightarrow M_{W}^{L}$ is the natural projection. Then these are glued together to define a well-defined global holomorphic vector field $\xi_{a}$ on 
$K_{M_{W}^{L}}$. We choose $\xi_{a}+\bar{\xi}_{a}$ as the Reeb field on $S_{W}^{L}$. However, we call $\xi_{a}$ also as the Reeb filed by abuse of terminology. Put

$$
z_{\alpha}^{+}:=\left(\tau_{\alpha}^{+}\right)^{-a} \zeta_{\alpha}^{+} \text {and } z_{\alpha}^{-}:=\left(\tau_{\alpha}^{-}\right)^{\frac{a}{1+2 a}} \zeta_{\alpha}^{-} .
$$

Then $\left(w_{\alpha}^{1}, w_{\alpha}^{2}, \ldots, w_{\alpha}^{n} ; z_{\alpha}^{+}\right)$and $\left(w_{\alpha}^{1}, w_{\alpha}^{2}, \ldots, w_{\alpha}^{n} ; z_{\alpha}^{-}\right)$are transverse holomorphic local coordinates on $\widetilde{U}_{\alpha}^{+}:=p^{-1}\left(U_{\alpha}^{+}\right)$and $\widetilde{U}_{\alpha}^{-}:=p^{-1}\left(U_{\alpha}^{-}\right)$, respectively, with respect to the Reeb field $\xi_{a}$, in view of the identities

$$
d z_{\alpha}^{+}\left(\xi_{a}\right)=0 \text { and } d z_{\alpha}^{-}\left(\xi_{a}\right)=0,
$$

where $p: S_{W}^{L} \longrightarrow M_{W}^{L}$ is the natural projection. Note that $z_{\alpha}^{+}$and $z_{\alpha}^{-}$ satisfy the following relation:

$$
z_{\alpha}^{+}=\left(\tau_{\alpha}^{+}\right)^{-a} \zeta_{\alpha}^{+}=\left(\tau_{\alpha}^{-}\right)^{-a}\left(\zeta_{\alpha}^{-}\right)^{-(1+2 a)}=\left(z_{\alpha}^{-}\right)^{-(1+2 a)} .
$$

For the natural projection $q: S_{W}^{L} \longrightarrow W$, the fiber $q^{-1}(w)$ over each $w \in U_{\alpha}$ has a transverse holomorphic structure defined by the transverse holomorphic coordinate $z_{\alpha}^{ \pm}$. Then on $q^{-1}(w)$,

$$
G:= \begin{cases}\left(\left|z_{\alpha}^{+}\right|^{-1}+\left|z_{\alpha}^{+}\right|^{\frac{1}{1+2 a}}\right)^{-2} \frac{\left|d z_{\alpha}^{+}\right|^{2}}{\left|z_{\alpha}^{+}\right|^{2}}, & \text { on } q^{-1}(w) \cap \widetilde{U}_{\alpha}^{+}, \\ (1+2 a)^{2}\left(\left|z_{\alpha}^{-}\right|^{1+2 a}+\left|z_{\alpha}^{-}\right|^{-1}\right)^{-2} \frac{\left|d z_{\alpha}^{-}\right|^{2}}{\left|z_{\alpha}^{-}\right|^{2}}, & \text { on } q^{-1}(w) \cap \widetilde{U}_{\alpha}^{+},\end{cases}
$$

defines a transverse Kähler metric, which is invariant under the standard $S^{1}$-action $z_{\alpha}^{+} \stackrel{t}{\longmapsto} t z_{\alpha}^{+}, t \in S^{1}$, for each $w \in U_{\alpha}$. By setting $x:=-2 \log \left|z_{\alpha}^{+}\right|$, we define

$$
v(x):=2 \log \left\{\exp \left(\frac{x}{2}\right)+\exp \left(-\frac{x}{2(1+2 a)}\right)\right\} .
$$

Then its derivative $v^{\prime}(x)$ defines a moment map whose image is the closed interval $\left[-\frac{1}{1+2 a}, 1\right]$.

\section{SASAKI-EINSTEIN METRICS ON $S_{W}^{L}$}

In this section, we construct an Sasaki-Einstein metric on $S_{W}^{L}$ by an argument as in [18]. For $a>-\frac{1}{2}$, define a polynomial $A_{a}(x)$ in $x$ by

$$
A_{a}(x):=-\int_{-\frac{1}{1+2 a}}^{x} s \prod_{k=1}^{n}\left(1+\mu_{k, a} s\right) d s .
$$

where $\mu_{k, a}:=\mu_{k}+a\left(1+\mu_{k}\right)$ for $k=1,2, \ldots, n$. Now, we assume that $A_{a}(1)=0$. Since $a>-\frac{1}{2}$, it follows from Assumption 1.3 that

$$
\begin{aligned}
& 0<A_{a}(x) \leqq A_{a}(0), \\
& \frac{A_{a}^{\prime}(x)}{x}<0,
\end{aligned}
$$


for $-\frac{1}{1+2 a}<x<1$. In particular, the rational function $\frac{A_{a}^{\prime}(x)}{x A_{a}(x)}$ is free from poles and zeros over the open interval $\left(-\frac{1}{1+2 a}, 1\right)$ and has a pole of order 1 at both $x=-\frac{1}{1+2 a}$ and $x=1$. Hence,

$$
B_{a}(x):=-\int_{0}^{x} \frac{A_{a}^{\prime}(s)}{s A_{a}(s)} d s
$$

is monotone increasing over the interval $\left(-\frac{1}{1+2 a}, 1\right)$ and moreover, $B_{a}$ maps $\left(-\frac{1}{1+2 a}, 1\right)$ diffeomorphically onto $\mathbb{R}$. Let

$$
B_{a}^{-1}: \mathbb{R} \longrightarrow\left(-\frac{1}{1+2 a}, 1\right)
$$

be the inverse function of $B_{a}:\left(-\frac{1}{1+2 a}, 1\right) \longrightarrow \mathbb{R}$, and define a $C^{\infty}$ functions $x_{a}(\rho)$ and $u_{a}(\rho)$ in $\rho \in \mathbb{R}$ by $x_{a}(\rho):=B_{a}^{-1}(\rho)$ and $u_{a}(\rho):=$ $-\log \left(A_{a}\left(x_{a}(\rho)\right)\right)$. Then $u_{a}^{\prime}(\rho)=x_{a}(\rho)$ and hence

$$
u_{a}^{\prime \prime}(\rho) \prod_{k=1}^{n}\left(1+\mu_{k, a} u_{a}^{\prime}(\rho)\right)=e^{-u_{a}(\rho)} .
$$

On $\widetilde{U}_{\alpha}^{+}$, we define $\rho_{\alpha}^{+}:=-\log \left|z_{\alpha}^{+}\right|^{2}-\log \left(\kappa_{\alpha}^{-a} h_{\alpha}^{1+a}\right)$ by setting

$$
\begin{aligned}
& \kappa_{\alpha}:=h_{K_{W}}\left(d w_{\alpha}^{1} \wedge \cdots \wedge d w_{\alpha}^{n}, d w_{\alpha}^{1} \wedge \cdots \wedge d w_{\alpha}^{n}\right), \\
& h_{\alpha}:=h\left(e_{\alpha}, e_{\alpha}\right),
\end{aligned}
$$

that is, $\exp \left(-\frac{\rho_{\alpha}^{+}}{2}\right)$ can be formally viewed as the norm of

$$
z_{\alpha}^{+}\left(\left(\frac{\partial}{\partial w_{\alpha}^{1}} \wedge \cdots \wedge \frac{\partial}{\partial w_{\alpha}^{n}}\right)^{a} \otimes e_{\alpha}^{1+a}\right)
$$

with respect to the Hermitian metric $h_{K_{W}}^{-a} \otimes h^{1+a}$ on $K_{W}^{-a} \otimes L^{1+a}$. Here $h_{K_{W}}$ denotes the Hermitian metric for $K_{W}$ induced by $\omega_{0}$. Then we have $\rho_{\alpha}^{+}=\rho_{\beta}^{+}$on $\widetilde{U}_{\alpha}^{+} \cap \widetilde{U}_{\beta}^{+}$. Now we consider the following transverse $(n+1, n+1)$-form $\Phi_{\alpha}$, with respect to $\xi_{a}$, on $\widetilde{U}_{\alpha}^{+}$:

$$
\Phi_{\alpha,+}:=\sqrt{-1}(n+1) \exp \left(-u_{a}\left(\rho_{\alpha}^{+}\right)\right)\left(q^{*} \omega_{0}\right)^{n} \wedge \frac{d z_{\alpha}^{+} \wedge d \overline{z_{\alpha}^{+}}}{\left|z_{\alpha}^{+}\right|^{2}} .
$$

Note that $\operatorname{Ric}\left(\omega_{0}\right)=\sqrt{-1} \bar{\partial} \partial \log \omega_{0}^{n}$ and that, for each fixed $w_{0} \in U_{\alpha}$, we can choose a local frame $e_{\alpha}$ for $L$ and a system $\left(w_{\alpha}^{1}, w_{\alpha}^{2}, \cdots, w_{\alpha}^{n}\right)$ of holomorphic local coordinates on $U_{\alpha}$ satisfying

$$
\begin{aligned}
& d\left(\kappa_{\alpha}^{-a} h_{\alpha}^{1+a}\right)\left(w_{0}\right)=0 \\
& \omega_{0}\left(w_{0}\right)=\sqrt{-1} \sum_{k=1}^{n} d w_{\alpha}^{k} \wedge d \overline{w_{\alpha}^{k}} \\
& \left(\sqrt{-1} \bar{\partial} \partial \log h_{\alpha}\right)\left(w_{0}\right)=\sqrt{-1} \sum_{k=1}^{n} \mu_{k} d w_{\alpha}^{k} \wedge d \overline{w_{\alpha}^{k}}
\end{aligned}
$$


Then, along $q^{-1}\left(w_{0}\right) \cap \widetilde{U}_{\alpha}^{+}$, we write $\omega_{\alpha,+}^{\mathrm{T}}:=\sqrt{-1} \bar{\partial} \partial \log \Phi_{\alpha,+}$ as a sum

$$
u^{\prime \prime}\left(\rho_{\alpha}^{+}\right) \frac{\sqrt{-1} d z_{\alpha}^{+} \wedge d \overline{z_{\alpha}^{+}}}{\left|z_{\alpha}^{+}\right|^{2}}+\sum_{k=1}^{n}\left\{\left(1+\mu_{k, a} u^{\prime}\left(\rho_{\alpha}^{+}\right)\right) \sqrt{-1} d w_{\alpha}^{k} \wedge d \overline{w_{\alpha}^{k}}\right\} .
$$

Since $a>-\frac{1}{2}$ and $-1<\mu_{k}<1(k=1,2, \ldots, n), \omega_{\alpha}^{\mathrm{T}}$ is a transverse Kähler form, with respect to $\xi_{a}$, on $\widetilde{U}_{\alpha}^{+} \backslash\left\{z_{\alpha}^{+}=0\right\}$. Furthermore, by (3.1), we have $\left(\omega_{\alpha,+}^{\mathrm{T}}\right)^{n+1}=\Phi_{\alpha,+}$. Therefore, $\omega_{\alpha}^{\mathrm{T}}$ defines a transverse Kähler-Einstein metric, with respect to $\xi_{a}$, on $\widetilde{U}_{\alpha}^{+} \backslash\left\{z_{\alpha}^{+}=0\right\}$. By an argument as in [18], the condition $A_{a}(1)=0$ implies that $\left\{\omega_{\alpha,+}^{\mathrm{T}} ;, \alpha \in A\right\}$ are glued together to define a well-defined global transverse KählerEinstein form $\omega^{\mathrm{T}}$ on $S_{W}^{L}$ with the Reeb field $\xi_{a}$.

Remark 3.2. $\Phi_{\alpha,+}$ is formally viewed as an Hermitian metric on $K_{W}^{-1} \otimes$ $\left(K_{W}^{a} \otimes L^{-(1+a)}\right)^{-1}=\left(K_{W} \otimes L^{-1}\right)^{-(1+a)}$. Then we put

$$
\begin{aligned}
& r:=\left|\tau_{\alpha}^{+}\right|\left\{(n+1) \exp \left(-u\left(\rho_{\alpha}^{+}\right)\right) \kappa_{\alpha}^{-1}\left|z_{\alpha}^{+}\right|^{-2}\right\}^{-\frac{1}{2(1+a)}}, \\
& \eta:=\left.(\sqrt{-1}(\bar{\partial}-\partial) \log r)\right|_{r=1} .
\end{aligned}
$$

Since $r=r\left(\tau_{\alpha}^{+}\right)$is regarded as the norm of

$$
\tau_{\alpha}^{+}\left(\left(\frac{\partial}{\partial w_{\alpha}^{1}} \wedge \cdots \wedge \frac{\partial}{\partial w_{\alpha}^{n}}\right) \otimes \frac{\partial}{\partial z_{\alpha}^{+}}\right)^{-\frac{1}{1+a}},
$$

with respect to the Hermitian metric $\left(\Phi_{\alpha,+}\right)^{-\frac{1}{1+a}}$ for $K_{W} \otimes L^{-1}, r$ defines a well-defined $C^{\infty}$ function on $K_{M_{W}^{L}}$, and in particular $S_{W}^{L}$ is identified with the submanifold of $K_{M_{W}^{L}}$ defined by the equation $r=1$. Moreover,

$$
g:=(\eta)^{2}+\frac{1}{1+a} g^{\mathrm{T}}
$$

is a Riemann metric on $S_{W}^{L}$ and $\eta$ is a contact form on $S_{W}^{L}$, where $g^{\mathrm{T}}$ is the transverse Kähler metric associated to $\omega^{\mathrm{T}}$. Furthermore, the fundamental form $\bar{\omega}$ of the cone metric $\bar{g}$ associated to $g$ is given by

$$
\bar{\omega}:=2 r d r \wedge \eta+\frac{r^{2}}{1+a} \omega^{\mathrm{T}} .
$$

In view of $d \eta=\frac{1}{1+a} \omega^{\mathrm{T}}$, we obtain $d \bar{\omega}=0$, and hence $\left(S_{W}^{L}, g\right)$ is a Sasaki manifold with the Reeb field $\xi_{a}$.

Now by Fact 1.1, we obtain the following criterion on the existence of Sasaki-Einstein metrics on $S_{W}^{L}$ :

Proposition 3.3. Under the Assumption 1.3, if

$$
A_{a}(1)=-\int_{-\frac{1}{1+2 a}}^{1} x \prod_{k=1}^{n}\left(1+\mu_{k, a} x\right) d x=0
$$

then $S_{W}^{L}$ admits a Sasaki-Einstein metric with the Reeb field $\xi_{a}$. 
Remark 3.5. In the special case $a=0$, we easily see that (3.4) is nothing but the condition (1.4) in the introduction.

Next, we shall show the existence of $a \in \mathbb{R}$ such that both $a>-\frac{1}{2}$ and $A_{a}(1)=0$ hold. We now put

$$
\begin{aligned}
& f(x ; a):=x \prod_{k=1}^{n}\left(1+\mu_{k, a} x\right), \\
& F(a):=\int_{-\frac{1}{1+2 a}}^{1} f(x ; a) d x \quad\left(=-A_{a}(1)\right) .
\end{aligned}
$$

Since $\lim _{a \rightarrow+\infty} F(a)=+\infty$ and $\lim _{a \rightarrow-\frac{1}{2}+0} F(a)=-\infty$, the continuity of $F$ allows us to find $a_{0}>-\frac{1}{2}$ such that $F\left(a_{0}\right)=0$. Moreover,

$$
F^{\prime}(a)=\int_{-\frac{1}{1+2 a}}^{1} \frac{\partial}{\partial a} f(x ; a) d x+\frac{-2}{(1+2 a)^{2}} f\left(-\frac{1}{1+2 a} ; a\right) .
$$

Note also that $\mu_{k, a}=\mu_{k}+a\left(1+\mu_{k}\right)$. Hence for $-\frac{1}{1+2 a} \leqq x \leqq 1$,

$$
\begin{aligned}
& \frac{\partial}{\partial a} f(x ; a)=x^{2} \sum_{j=1}^{n}\left\{\left(1+\mu_{j}\right) \prod_{k \neq j}\left(1+\left\{\mu_{k}+a\left(1+\mu_{k}\right)\right\} x\right)\right\} \geqq 0, \\
& f\left(-\frac{1}{1+2 a} ; a\right)=-\left(\frac{1}{1+2 a}\right)^{n+1} \prod_{k=1}^{n}\left\{(1+a)\left(1-\mu_{k}\right)\right\}<0 .
\end{aligned}
$$

Now in the expression of $F^{\prime}(a)$, the first term is nonnegative and the second term is positive. Therefore $F^{\prime}(a)>0$. Hence we obtain:

Lemma 3.6. Under the Assumption 1.3, there exists a unique real number $a_{0}>-\frac{1}{2}$ such that $F\left(a_{0}\right)=0$.

Therefore, by Proposition 3.3 and Lemma 3.6, if Assumption 1.3 is satisfied, then $S_{W}^{L}$ always admits an Sasaki-Einstein metric with the Reeb field $\xi_{a_{0}}$. On the other hand, in view of [9], [11](see also [26]), we now conclude that $K_{M_{W}^{L}}$ admits a complete Ricci-flat Kähler metric in every Kähler class. The proof of Theorem 1.5 is now complete.

\section{ExAmples}

In this section, we shall give a couple of examples of Sasaki-Einstein manifolds as an application of Theorem 1.5.

Example 4.1. We first put

$$
\begin{aligned}
& W:=\prod_{i=1}^{l} \mathbb{P}^{n_{i}}(\mathbb{C}), \\
& L:=\bigotimes_{i=1}^{l} p_{i}^{*}\left(\mathcal{O}_{\mathbb{P}^{n_{i}(\mathbb{C})}}\left(\nu_{i}\right)\right),
\end{aligned}
$$


where $p_{i}: W \longrightarrow \mathbb{P}^{n_{i}}(\mathbb{C})$ is the natural projection to the $i$-th factor $(i=1,2, \ldots, l)$. In view of the isomorphism $K_{\mathbb{P}^{k}(\mathbb{C})}^{-1} \cong \mathcal{O}_{\mathbb{P}^{k}(\mathbb{C})}(k+1)$, if

$$
-\left(n_{i}+1\right)<\nu_{i}<n_{i}+1, \quad(i=1,2, \ldots, l),
$$

then the pair $(W, L)$ satisfies Assumption 1.3. Hence by Theorem 1.5, $S_{W}^{L}$ admits a Sasaki-Einstein metric, though this is toric. Then $F(a)$ in Section 3 is given by

$$
F(a)=\int_{-\frac{1}{1+2 a}}^{1} x \prod_{i=1}^{l}\left(1+\left\{\frac{\nu_{i}}{n_{i}+1}+a\left(1+\frac{\nu_{i}}{n_{i}+1}\right)\right\} x\right)^{n_{i}} d x .
$$

For instance, we consider the simplest case, that is, $W=\mathbb{P}^{1}(\mathbb{C})$ and $L=\mathcal{O}_{\mathbb{P}^{1}(\mathbb{C})}(1)$. In this case, $M_{W}^{L}$ is a del Pezzo surface obtained from $\mathbb{P}^{2}(\mathbb{C})$ by blowing up one point, and we see the irregularity of $\left(S_{W}^{L}, \xi_{a_{0}}\right)$ by

$$
a_{0}=\frac{-5+\sqrt{13}}{12}
$$

Example 4.2. Next, let $W:=\operatorname{Gr}(k, p)$ be the complex Grassmannian manifold of all $p$-dimensional subspaces of $\mathbb{C}^{k}$, which is a complex manifold of dimension $p(k-p)$. Then there exists an ample line bundle $A(k, p)$ over $\operatorname{Gr}(k, p)$ such that $K_{\mathrm{Gr}(k, p)}^{-1} \cong A(k, p)^{k}$ (see for instance [24, p. 205]). We put $L:=A(k, p)^{\nu}$. If $-k<\nu<k$, then the pair $(W, L)$ satisfies Assumption 1.3. Hence by Theorem 1.5, $S_{W}^{L}$ admits a Sasaki-Einstein metric, and if $2 \leqq p \leqq k-2$, then $S_{W}^{L}$ is non-toric.

Example 4.3. Let $\mathcal{M}_{n}$ be the moduli space of smooth hypersurfaces of degree $n$ in $\mathbb{P}^{n+1}(\mathbb{C})$. For the Fermat type hypersurface

$$
W_{0}:=\left\{\left[X_{0}: \ldots: X_{n+1}\right] ;\left(X_{0}\right)^{n}+\cdots+\left(X_{n+1}\right)^{n}=0\right\} \in \mathcal{M}_{n},
$$

a theorem of Tian [25] shows that $W_{0}$ admits a Kähler-Einstein metric, and in particular

$$
\mathcal{M}_{n}^{\mathrm{KE}}:=\left\{W \in \mathcal{M}_{n} ; W \text { admits a Kähler-Einstein metric. }\right\}
$$

is a non-empty open subset of $\mathcal{M}_{n}$. For every $W \in \mathcal{M}_{n}^{\mathrm{KE}}$, we have $\left.K_{W} \cong \mathcal{O}_{\mathbb{P}^{n+1}(\mathbb{C})}(-2)\right|_{W}$ by adjunction formula. Put $L:=\left.\mathcal{O}_{\mathbb{P}^{n+1}(\mathbb{C})}(1)\right|_{W}$. Then the pair $(W, L)$ satisfies Assumption 1.3, and Theorem 1.5 shows that $S_{W}^{L}$ admits a Sasaki-Einstein metric. If $n=3, W$ is a wellknown cubic threefold, and in this case by [7, Theorem 13.12], $W$ is not birationally equivalent to $\mathbb{P}^{3}(\mathbb{C})$, and $S_{W}^{L}$ is again non-toric.

\section{REFERENCES}

[1] K. Cho, A. Futaki and H. Ono, Uniqueness and examples of compact toric Sasaki-Einstein metrics, Commun. Math. Phys. 277 (2008), pp. 439-458.

[2] C. P. Boyer and K. Galicki, 3-Sasakian manifolds, Surveys Diff. Geome. 7 (1999), pp. 123-184.

[3] C. P. Boyer and K. Galicki, A note on toric contact geometry, J. Geome. Phys. 35 (2000), pp. 288-298. 
[4] C. P. Boyer and K. Galicki, "Sasakian Geometry", Oxford Mathematical Monographs, Oxford University Press, Oxford, 2008.

[5] C. P. Boyer, K. Galicki and J. Kollár, Einstein metrics on spheres, Ann. of Math. 162 (2005), pp. 557-580.

[6] C. P. Boyer, K. Galicki and S. R. Simanca, Canonical Sasakian metrics, Comm. Math. Phys. 279 (2008), pp. 705-733.

[7] C. H. Clemens and P. A. Griffiths, The intermediate Jacobian of the cubic threefold, Ann. of Math. 95 (1972), pp. 281-356.

[8] A. Futaki, An obstruction to the existence of Kähler Einstein metrics, Invent. Math. 73 (1983), pp. 437-443.

[9] A. Futaki, Momentum construction on Ricci-flat Kähler cones, Tohoku Math. J. 63 (2011), pp. 21-40.

[10] A. Futaki, H. Ono and G. Wang, Transverse Kähler Geometry of Sasaki manifolds and toric Sasaki-Einstein manifolds, J. Differential Geom. 83 (2009), pp. 585-635.

[11] R. Goto, Calabi-Yau structures and Einstein-Sasakian structures on crepant resolutions of isolated singularities, preprint, arXiv:0906.5191.

[12] Y. Hashimoto, M. Sakaguchi and Y. Yasui, Sasaki-Einstein twist of Kerr-AdS black holes, Phys. Lett. B 600 (2004), pp. 270-274.

[13] G. R. Jensen, Einstein metrics on principal fibre bundles, J. Differential Geom. 8 (1973), pp. 599-614.

[14] S. Kobayashi, Topology of positively pinched Kaehler manifolds, Tohoku Math. J. 15 (1963), pp. 121-139.

[15] N. Koiso, On rotationally symmetric Hamilton's equation for Kähler-Einstein metrics, in "Kähler metric and moduli spaces", Adv. Stud. in Pure Math. 18-I, Kinokuniya and Academic Press, Tokyo and Boston, 1990, pp. 327-337.

[16] N. Koiso and Y. Sakane, Non-homogeneous Kähler-Einstein metrics on compact complex manifolds, in "Curvature and topology of Riemannian manifolds", Lecture Notes in Math. 1201, Springer-Verlag, Berlin, Heidelberg, New York, 1986, pp. $165-179$.

[17] N. Koiso and Y. Sakane, Non-homogeneous Kähler-Einstein metrics on compact complex manifolds, II, Osaka J. Math. 25 (1988), pp. 933-959.

[18] T. Mabuchi, Einstein-Kähler forms, Futaki invariants and convex geometry on toric Fano varieties, Osaka J. Math. 24 (1987), pp. 705-737.

[19] D. Martelli and J. Sparks, Toric Sasaki-Einstein metrics on $S^{2} \times S^{3}$, Phys. Lett. B 621 (2005), pp. 208-212.

[20] D. Martelli and J. Sparks, Toric geometry, Sasaki-Einstein manifolds and a new infinite class of AdS/CFT duals, Comm. Math. Phys. 262 (2006), pp. 51-89.

[21] D. Martelli, J. Sparks and S. T. Yau, The geometric dual of a-maximisation for toric Sasaki-Einstein manifolds, Comm. Math. Phys. 268 (2006), pp. 39-65.

[22] D. Martelli, J. Sparks and S. T. Yau, Sasaki-Einstein manifolds and volume minimisation, Comm. Math. Phys. 280 (2008), pp. 611-673.

[23] Y. Sakane, Example of compact Einstein Kähler manifolds with positive Ricci tensor, Osaka J. Math. 23 (1986), pp. 585-616.

[24] M. Takeuchi, Homogeneous Kähler submanifolds in complex projective spaces, Janan. J. Math. 4 (1978), pp. 171-219.

[25] G. Tian, On Kähler-Einstein metrics on certain Kähler manifolds with $C_{1}(M)>0$, Invent. Math. 89 (1987), pp. 225-246.

[26] C. van Coevering, Ricci-flat Kähler metrics on crepant resolutions of Kähler cones, Math. Ann. 347 (2010), pp. 581-611. 
[27] M. Y. Wang and W. Ziller, Einstein metrics on principal torus bundles, J. Differential Geom. 31 (1990), pp. 215-248.

[28] X.-J. Wang and X. Zhu, Kähler-Ricci solitons on toric manifolds with positive first Chern class, Adv. Math. 188 (2004), pp. 87-103.

*Department of Mathematics, Osaka University, Toyonaka, Osaka 560-0043, JAPAN

E-mail address: mabuchi@math.sci.osaka-u.ac.jp

**School of Mathematics And Physics, College of Science And EnGineEring, KanaZawa University, KaKuma-Machi, KanaZaWA, 920-1192, JAPAN

E-mail address: yasunaka@kenroku.kanazawa-u.ac.jp 